\title{
Comparison of femoral block (FB), femoral + sciatic block by posterior route (FCB) and epidural block (EB) in the control of acute postoperative pain after knee arthroplasty
}

\author{
${ }^{1}$ Sara López Palanca, ${ }^{1}$ Lucas Rovira Soriano, ${ }^{2}$ Beatriz Bellón López de Antón Bueno, ${ }^{1}$ José De Andrés Ibáñez \\ ${ }^{1}$ Anesthesia and Intensive Care. General Hospital of Valencia. Valencia. Spain. ${ }^{2}$ Anesthesia and Intensive Care. General Hospital of Villarrobledo. Albacete. Spain
}

\section{Background:}

- Arthrosis is a degenerative disease that damages the cartilage, bone and synovial membrane of the joint, and that is accompanied by pain and inability for physical activity.

- Total knee arthroplasty (TKA) is a surgical procedure that consists of replacing the injured or degenerated joint surfaces with an artificial joint (prosthesis). This surgery aims to eliminate pain, restore joint mobility and improve the quality of life of affected patients

The current approach to postoperative pain in major knee surgery is based on a multimodal analgesia model, which consists of the association of different analgesic techniques that act at different levels of the pain pathways. The current recommendations are the association of acetaminophen / NSAID + regional technique (epidural or peripheral nerve blocks), relieving the use of opiates as rescue analgesia or in situations in which locoregional techniques are contraindicated, refused by the patient or not its realization was possible.

The aim of our study was to compare the efficacy and safety of different analgesic regional techniques for the control of DAP such as femoral block (FB), femoral block combined with posterior sciatic block (FCB), and epidural block. (EB) after ATR surgery.
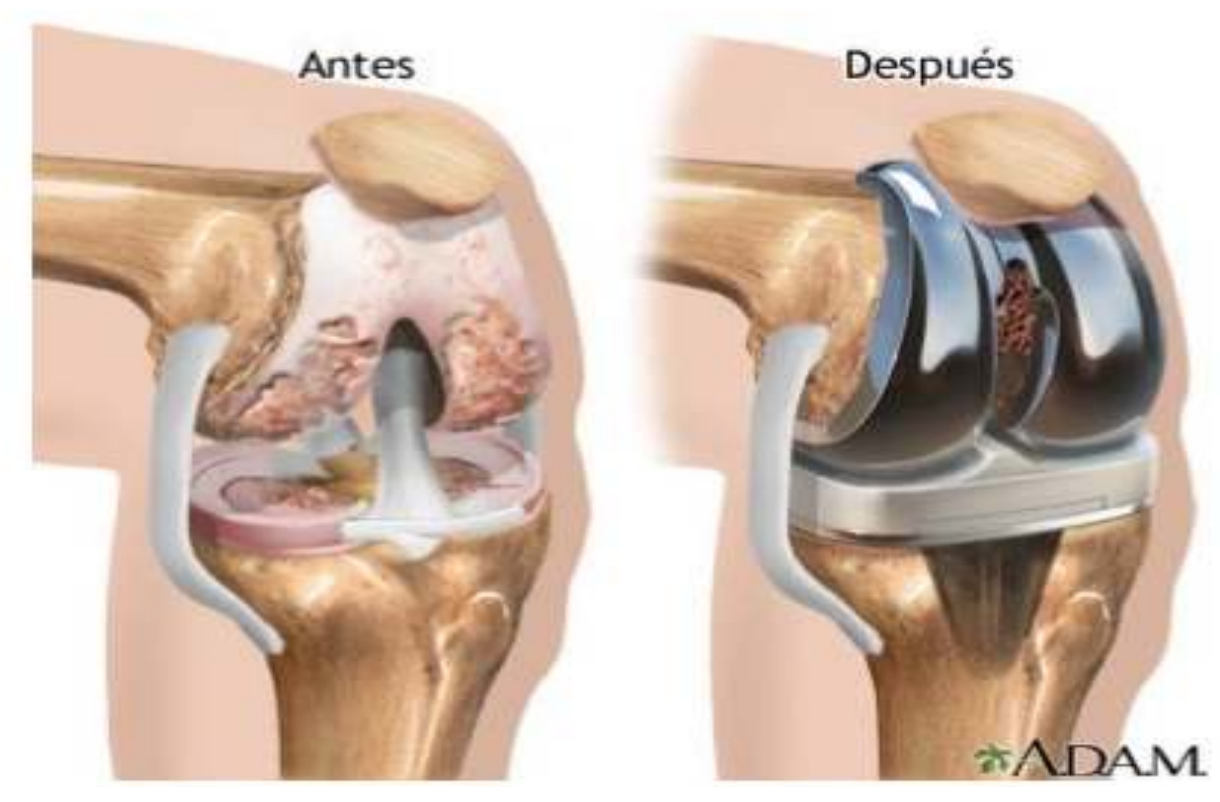

\section{Materials and methods:}

A prospective observational study was conducted in which 30 patients undergoing TKA from June 2016 to May 2017 were included. In all patients, the anesthetic technique used in the operating room was an intradural anesthesia with $0.5 \%$ hyperbaric bupivacaine between 11 and $13 \mathrm{mg}+$ fentaline $10-20 \mathrm{mcg}$. After the surgical intervention and once the anesthetic motor block was recovered, the epidural infusion was started through the placed catheter or the peripheral nerve block was performed by neurostimulation and echoguiding. For this, 3 groups of patients were divided to compare the different postoperative analgesic techniques to be performed, such as the femoral block (FB), the femoral block combined with posterior sciatic block (FCB), and the epidural block (EB):

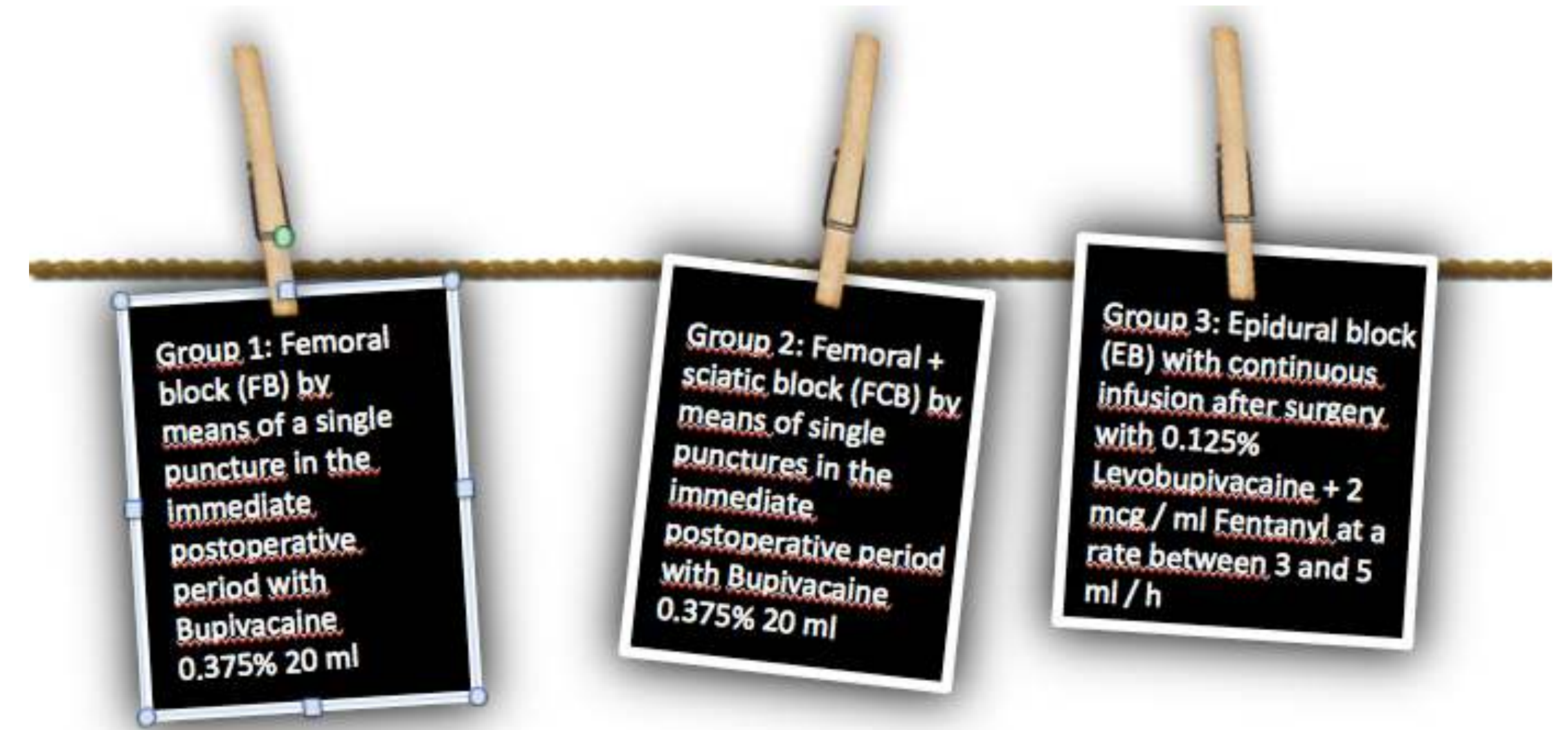

\section{Conclusions:}

There is still no consensus based on the evidence on the management of postoperative pain. The ideal analgesic technique in the postoperative period of TKA would be one that provides an important control of pain, with minimal side effects, with the least possible impact on motor function in order to facilitate early postoperative mobilization, optimal rehabilitation and get early hospital discharge.

Peripheral nerve blocks are an equally effective alternative in terms of the quality of analgesia provided in the postoperative period with the advantage of producing fewer side effects. In our study, the association of a sciatic block to the femoral block is related to a lower painful intensity and lower opioid consumption in the postoperative period of TKA compared to the isolated femoral block, since this leaves the posterior part of the knee uncovered. In addition, these regional blockades, in turn, have fewer adverse events and are less severe than the epidural block group 